\title{
Cross-platform meta-analysis of multiple gene expression profiles identifies novel expression signatures in acquired anthracycline-resistant breast cancer
}

\author{
YOUNG SEOK LEE ${ }^{1}$, SEUNG WON RYU ${ }^{1}$, SE JONG BAE ${ }^{1}$, TAE HWAN PARK ${ }^{3}$, \\ $\mathrm{KANG} \mathrm{KWON}^{4}$, YUN HEE NOH ${ }^{1}$ and SUNG YOUNG KIM ${ }^{1,2}$ \\ ${ }^{1}$ Department of Biochemistry and ${ }^{2}$ Research Institute of Medical Science, Konkuk University School of Medicine, \\ Seoul 143-701; ${ }^{3}$ Institute for Human Tissue Restoration, Department of Plastic and Reconstructive Surgery, \\ College of Medicine, Yonsei University, Seoul 120-749; ${ }^{4}$ Pusan National University \\ School of Korean Medicine, Pusan 609-735, Republic of Korea
}

Received December 1, 2014; Accepted February 2, 2015

DOI: 10.3892/or.2015.3810

\begin{abstract}
Anthracyclines are among the most effective and commonly used chemotherapeutic agents. However, the development of acquired anthracycline resistance is a major limitation to their clinical application. The aim of the present study was to identify differentially expressed genes (DEGs) and biological processes associated with the acquisition of anthracycline resistance in human breast cancer cells. We performed a meta-analysis of publically available microarray datasets containing data on stepwise-selected, anthracycline-resistant breast cancer cell lines using the RankProd package in R. Additionally, the gene ontology (GO) and Kyoto Encyclopedia of Genes and Genomes (KEGG) databases were used to analyze GO term enrichment and pathways, respectively. A protein-protein interaction (PPI) network was also generated using Cytoscape software. The meta-analysis yielded 413 DEGs related to anthracycline resistance in human breast cancer cells, and 374 of these were not involved in individual DEGs. GO analyses showed the 413 genes were enriched with terms such as 'response to steroid metabolic process', 'chemical stimulus', 'external stimulus', 'hormone stimulus', 'multicellular organismal process', and 'system development'. Pathway analysis revealed significant pathways including steroid hormone biosynthesis, cytokine-cytokine receptor interaction, drug metabolism-cytochrome P450, metabolism of xenobiotics by cytochrome $\mathrm{P} 450$, and arachidonic acid metabolism. The PPI network indicated that proteins encoded by TRIM29, VTN, CCNA1, and karyopherin a 5 (KPNA5) participated in a significant number of interactions. In conclu-
\end{abstract}

Correspondence to: Professor Sung Young Kim, Department of Biochemistry, Konkuk University School of Medicine, 1 Hwayangdong, Seoul 143-701, Republic of Korea

E-mail: palelamp@kku.ac.kr

Key words: meta-analysis, gene expression, drug resistance, anthracycline, breast cancer sion, our meta-analysis provides a comprehensive view of gene expression patterns associated with acquired resistance to anthracycline in breast cancer cells, and constitutes the basis for additional functional studies.

\section{Introduction}

Anthracyclines such as doxorubicin and epirubicin are chemotherapeutic drugs used to effectively treat various types of cancer, including leukemia and breast, ovarian, uterine, and lung cancers (1). Despite being introduced over 30 years ago, anthracyclines remain part of the gold standard chemotherapy for breast cancer (2). However, a significant number of breast cancer patients acquire resistance to these drugs during chemotherapy $(3,4)$. Drug resistance can be classified into two main categories: intrinsic drug resistance, in which previously untreated cancer cells are inherently insensitive to the chemotherapeutic drugs; and acquired drug resistance (ADR), in which the cancer cells become insensitive after drug exposure $(5,6)$. The mechanisms of drug resistance have been intensively studied with the aim of overcoming this major obstacle in chemotherapy, and, although the exact mechanisms of ADR remain unclear, numerous theories have been proposed. One mechanism that may underlie acquired resistance to anthracycline is the active cellular extrusion of drugs by the overexpression of multidrug resistance protein 1 (MDR1) (7). $M D R 1$, also known as P-glycoprotein 1 (permeability glycoprotein, abbreviated as P-gp or Pgp) or ATP-binding cassette (ABC) sub-family B member $1(A B C B 1)$, acts as an efflux pump for various xenobiotics such as toxins or drugs. While in vitro studies have demonstrated the efficacy of certain $M D R l$ inhibitors, in clinical studies, these compounds have yet to show a consistent advantage $(8,9)$. Other than drug efflux pumps, mechanisms that may contribute to anthracycline resistance include: changes in intracellular drug distribution, apoptotic and DNA repair responses and alteration of topoisomerase II, which is the major cellular target of anthracyclines $(10,11)$. However, the validity of these particular mechanisms of action remains to be ascertained. 
Table I. Characteristics of individual studies analyzed in the meta-analysis.

\begin{tabular}{|c|c|c|c|c|c|}
\hline \multirow[b]{2}{*}{ GEO dataset } & \multicolumn{2}{|c|}{$\begin{array}{l}\text { No. of } \\
\text { samples }\end{array}$} & \multirow[b]{2}{*}{ Drug } & \multirow[b]{2}{*}{ Cell line } & \multirow[b]{2}{*}{ Platform } \\
\hline & $\mathrm{PC}$ & AR & & & \\
\hline GSE24460 & 2 & 2 & Doxorubicin & MCF-7 & Affymetrix human genome U133A array \\
\hline GSE3926 & 1 & 2 & Doxorubicin & MCF-7 & Affymetrix human genome U133A array \\
\hline GSE54326 & 12 & 12 & Epirubicin & $\begin{array}{l}\text { MDA-MB-231, MCF-7, } \\
\text { SKBR-3, ZR-75-1 }\end{array}$ & Illumina human HT-12 V4.0 expression beadchip \\
\hline
\end{tabular}

GEO, gene expression omnibus; GSE, gene expression series; PC, parental control; AR, anthracycline-resistant.

ADR is multifactorial because it involves host factors, various molecular events, and numerous genetic changes (12). In developing targeted therapeutic strategies to overcome drug resistance, it is essential to understand the basic genetic changes associated with acquisition of drug resistance. High-throughput gene sequencing technologies, such as microarrays, are widely used to comprehensively analyze gene expression, and to detect mutations and single-nucleotide polymorphisms (13). By applying these technologies, investigators have improved their understanding of the cellular and molecular changes that occur during the development of ADR in breast cancer $(14,15)$. Previous studies have provided lists of differentially expressed genes (DEGs); however, findings tend to be inconsistent across studies due to small sample sizes, and differences in sample quality, laboratory protocol, platform, and analytical technique (16). In order to overcome the limitations presented by these inconsistencies, it is possible to take a systematic approach and perform integrated analyses of multiple microarray datasets.

Interest in using integrated analysis to investigate multiple independent microarray datasets has been on the increase (17). Accumulating evidence has shown that meta-analysis increases the statistical power of expression profiling and enables an assessment of between-study heterogeneity, which may lead to more robust and reliable gene signatures $(17,18)$. To the best of our knowledge, a meta-analysis focusing on data for acquired resistance to anthracycline in breast cancer has yet to be performed. Therefore, in the present study, we performed the first cross-platform meta-analysis of multiple gene expression profiles taken from various independent studies with the aim of identifying novel candidate genes and biological processes that are involved in acquired anthracycline resistance, and overcoming the limitations presented by inconsistencies in individual studies.

\section{Materials and methods}

Extraction of eligible microarray datasets containing data on anthracycline-resistant breast cancer cell lines. Gene expression studies related to acquired anthracycline resistance in breast cancer were collected in July 2014 by searching the PubMed database, NCBI Gene Expression Omnibus (GEO) (available at: http://www.ncbi.nlm.nih.gov/geo/), and ArrayExpress (AE) (available at: http://www.ebi.ac.uk/arrayexpress/). When searching these resources, the following keywords and their combinations were used: 'anthracycline', 'drug resistance', 'breast cancer', and 'gene expression'. Two independent reviewers extracted data from the original studies. Any discrepancies between the reviewer's data were resolved either by consensus or a third reviewer. Inclusion criteria for the study were: i) gene expression profiling of stepwise-selected, anthracycline-resistant, derivative breast cancer cell lines; and ii) sufficient data and the correct platform to facilitate the meta-analysis. We retained only those original experimental articles in which gene expression profiles of stepwise-selected, anthracycline-resistant, derivative breast cancer cell lines were analyzed relative to parental control cells. Non-human data, review articles, and integrated analysis of expression profiles were also excluded from the meta-analysis. The following information was extracted from each selected study: GEO accession number, platform and sample type, and gene expression data.

Meta-analysis of gene expression in multiple microarray datasets. We used the meta-analysis of the gene expression profiles in the selected microarray datasets to identify DEGs. Prior to processing of data, all the gene and probe IDs were annotated as Entrez IDs for consistency, and intensity values were $\log 2$ transformed and normalized in order that their mean and unit variance was zero. A meta-analysis was performed using rank product methods (RankProd package in R) implemented in the web-based INMEX program. RankProd (developed from the non-parametric rank product method) was used to apply a statistically rigorous algorithm, which included biological intuition of fold-change (FC) criteria and determined the ranks of the DEGs based on FC scores in all possible pairwise comparisons, to the integrated datasets. With the RankProd algorithm, genes that were consistently identified as up- or downregulated DEGs in whole datasets were assigned a higher rank depending on their $\mathrm{P}$-value and $\mathrm{FC}$ level in a given number of replicates multiplied across the given datasets, and these were considered the most significantly regulated DEGs. The expression profiles of DEGs across different datasets/conditions were visualized as heat maps by implementing the 'Pattern Extractor' tool.

Functional and pathway enrichment analyses of DEGs. To investigate the cellular function of DEGs, we performed a gene ontology (GO) enrichment analysis based on the GO database 
Table II. The top 20 most strongly up- or downregulated DEGs by meta-analysis.

\begin{tabular}{|c|c|c|c|c|}
\hline Enterz ID & Gene symbol & Average log FC & P-value & Gene name \\
\hline \multicolumn{5}{|c|}{$\begin{array}{l}\text { Upregulated } \\
\text { genes }\end{array}$} \\
\hline 23650 & TRIM29 & -11.5579 & $<1.0 \mathrm{E}-5$ & Tripartite motif containing 29 \\
\hline 5797 & PTPRM & -11.2551 & & Protein tyrosine phosphatase, receptor type, $\mathrm{M}$ \\
\hline 23136 & EPB41L3 & -10.6225 & & Erythrocyte membrane protein band 4.1-like 3 \\
\hline 7448 & VTN & -9.83827 & & Vitronectin \\
\hline 5243 & $\mathrm{ABCB} 1$ & -9.73049 & & ATP-binding cassette, sub-family B (MDR/TAP), member 1 \\
\hline 8581 & LY6D & -6.59411 & & Lymphocyte antigen 6 complex, locus D \\
\hline 56999 & ADAMTS9 & -6.5461 & & ADAM metallopeptidase with thrombospondin type 1 motif, 9 \\
\hline 4939 & OAS2 & -5.92265 & & 2'-5'-oligoadenylate synthetase 2,69/71 kDa \\
\hline 57007 & CXCR7 & -5.88834 & & Atypical chemokine receptor 3 \\
\hline 57016 & AKR1B10 & -5.12808 & & Aldo-keto reductase family 1 , member B 10 \\
\hline 972 & CD74 & -8.34455 & 0.00050 & $\begin{array}{l}\text { CD74 molecule, major histocompatibility complex, class II } \\
\text { invariant chain }\end{array}$ \\
\hline 54894 & RNF43 & -7.23015 & 0.00053 & Ring finger protein 43 \\
\hline 6590 & SLPI & -6.86764 & 0.00056 & Secretory leukocyte peptidase inhibitor \\
\hline 7124 & TNF & -7.69007 & 0.00059 & Tumor necrosis factor \\
\hline 10964 & IFI44L & -6.05839 & 0.00063 & Interferon-induced protein 44-like \\
\hline 6707 & SPRR3 & -5.51409 & 0.00067 & Small proline-rich protein 3 \\
\hline 79132 & DHX58 & -7.722 & 0.00071 & DEXH (Asp-Glu-X-His) box polypeptide 58 \\
\hline 7078 & TIMP3 & -6.28697 & 0.00077 & TIMP metallopeptidase inhibitor 3 \\
\hline 9429 & ABCG2 & -6.67666 & 0.00083 & ATP-binding cassette, sub-family G (white), member 2 \\
\hline 4973 & OLR1 & -7.30092 & 0.00091 & Oxidized low-density lipoprotein (lectin-like) receptor 1 \\
\hline \multicolumn{5}{|c|}{$\begin{array}{l}\text { Downregulated } \\
\text { genes }\end{array}$} \\
\hline 767 & CA8 & 10.97236 & $<1.0 \mathrm{E}-5$ & Carbonic anhydrase VIII \\
\hline 55130 & ARMC4 & 10.22814 & & Armadillo repeat containing 4 \\
\hline 6641 & SNTB1 & 9.843391 & & $\begin{array}{l}\text { Syntrophin, } \beta 1 \\
\text { (dystrophin-associated protein A1, basic component 1) }\end{array}$ \\
\hline 8900 & CCNA1 & 9.58363 & & Cyclin A1 \\
\hline 3841 & KPNA5 & 9.107488 & & Karyopherin $\alpha 5$ (importin $\alpha 6$ ) \\
\hline 64208 & POPDC3 & 8.982291 & & Popeye domain containing 3 \\
\hline 7552 & ZNF711 & 8.752859 & & Zinc finger protein 711 \\
\hline 2346 & FOLH1 & 8.277195 & & Folate hydrolase (prostate-specific membrane antigen) 1 \\
\hline 117247 & SLC16A10 & 8.21847 & & $\begin{array}{l}\text { Solute carrier family } 16 \text { (aromatic amino acid transporter), } \\
\text { member } 10\end{array}$ \\
\hline 1809 & DPYSL3 & 8.109953 & & Dihydropyrimidinase-like 3 \\
\hline 7033 & TFF3 & 6.606418 & & Trefoil factor 3 (intestinal) \\
\hline 563 & AZGP1 & 5.309637 & & $\alpha$-2-glycoprotein 1 , zinc-binding \\
\hline 79679 & VTCN1 & 3.976014 & & V-set domain containing T-cell activation inhibitor 1 \\
\hline 54504 & CPVL & 2.726046 & & Carboxypeptidase, vitellogenic-like \\
\hline 26154 & ABCA12 & 7.400289 & 0.00067 & ATP-binding cassette, sub-family A (ABC1), member 12 \\
\hline 26047 & CNTNAP2 & 2.945366 & 0.00125 & Contactin-associated protein-like 2 \\
\hline 23493 & HEY2 & 3.766967 & 0.00167 & $\begin{array}{l}\text { Hes-related family bHLH transcription factor with YRPW } \\
\text { motif } 2\end{array}$ \\
\hline 6578 & SLCO2A1 & 5.874261 & 0.00176 & Solute carrier organic anion transporter family, member $2 \mathrm{~A} 1$ \\
\hline 241 & ALOX5AP & 3.967401 & 0.00200 & Arachidonate 5-lipoxygenase-activating protein \\
\hline 89874 & SLC25A21 & 6.886683 & 0.00211 & $\begin{array}{l}\text { Solute carrier family } 25 \text { (mitochondrial oxoadipate carrier), } \\
\text { member } 21\end{array}$ \\
\hline
\end{tabular}

FC, fold change. 
Table III. The top 5 enriched terms in biological process of GO analysis.

\begin{tabular}{|c|c|c|c|}
\hline GO ID & Term & P-value & Genes \\
\hline GO:0008202 & $\begin{array}{l}\text { Steroid } \\
\text { metabolic } \\
\text { process }\end{array}$ & $2.15 \mathrm{E}-11$ & $\begin{array}{l}\text { AKR1B10; TNF; UGT2B15; LRP2; NPC1L1; CYP3A5; CELA3A; ESR1; } \\
\text { AKR1C3; ACADL; LCAT; HSD3B1; SOAT2; LMF1; UGT2B17; HSD17B1; } \\
\text { EPHX2; HSD17B14; SLCO1B3; HSD17B2; STS; NR5A2; MT3; SULT1B1; } \\
\text { TFCP2L1; APOA1; HSD3B2; DKK3; LIPC; CACNA1H }\end{array}$ \\
\hline GO:0042221 & $\begin{array}{l}\text { Response } \\
\text { to chemical } \\
\text { stimulus }\end{array}$ & $1.28 \mathrm{E}-10$ & $\begin{array}{l}\text { VTN; ABCB1; PTPRM; CXCR7; LY6D; OAS2; DPYSL3; OLR1; ABCG2; } \\
\text { TIMP3; TNF; CD74; KPNA5; UGT2B15; ACP5; ALOX5AP; CCL20; POU3F2; } \\
\text { TFF1; MYL9; BRCA2; LRP2; DLG4;ALDH3A1; LY96; IL1R1; CYP4F8; } \\
\text { CYP3A5; CYP3A7; NTF3; AFF3; CCL16; TH; SLC6A14; ESR1;AKR1C3; } \\
\text { GCKR; LCAT; TESC; SPARC;S100A12; HSPB7; GHR; NGF; PTGS2; } \\
\text { COLEC12; FOXA1; BMP7; VN1R1;KRT13; MGMT; SLC1A3; CIITA; } \\
\text { RARRES2; GATM; KYNU; PDE1C; PTGER2; PLK3; CA2; PDE3B;PSMB8; } \\
\text { NRAS; CPB2; LHX2; GNB3; FGFBP1; CALCR; NPPB; EPHX2; CX3CL1; GIP; } \\
\text { LMO2; NNMT; MAP1B; GH2; GSTM3; CUX2; EBI3; PGR; SERPINA1; FMO3; } \\
\text { IFIT3; HTR2B; NRP2; PLA2G7; HERC5; HSD17B2; FADS1; LUM; STS; } \\
\text { NRCAM; HTR1B; MT3; SULT1B1; IRAK3; MICB; ABCB4; FES; PDGFRB; } \\
\text { MAT1A; GNAI1; ARTN; APOA1; S100A7; IL6; FZD5; IL15RA; RAC2; } \\
\text { CACNA1H; REN; CD14; ACSL5; SEMA3A; TRPC6; MPP1; TRPM6; GPR77 }\end{array}$ \\
\hline
\end{tabular}

GO:0050896 Response to stimulus

GO:0009605 Response to external stimulus
7.84E-09 CA8; VTN; ABCB1; PTPRM; CXCR7;LY6D; OAS2; DPYSL3; OLR1; ABCG2; TIMP3; DHX58; SPRR3;IFI44L; TFF3; TNF; RNF43; CD74; KPNA5; AZGP1; FSTL1; IL32; CEACAM6; UGT2B15;CNTNAP2; ACP5; HEY2; ALOX5AP; CCL20; NPM1; POU3F2; TFF1; CLEC1A; ABCA4; MYL9; BRCA2; LRP2; MAGEA1; DLG4; ALDH3A1; GUCY1B3; PRRX2; GP2; CDH2; LY96; CPQ; IL1R1; CYP4F8; STRA6; NEDD9; LGALS9; CYP3A5; CYP3A7; NTF3; AFF3; GPX2; CCL16; TH; NT5E; SLC6A14; ITGA6; NR2F1;ESR1; CD3D; HRK; SRGN; CD19; AKR1C3; GCKR; LCAT; TAAR5; TESC; CD33; IQGAP2; CSPG4; SPARC; S100A12; CUL3; TLE4; INSL4; HSPB7; GHR; NGF; CEACAM1; PTGS2; COLEC12; FOXA1; DTX3; BMP7; SIRPA; VN1R1; KRT13; MGMT; BFSP2; SLC1A3; CDC42EP3; FGD1;CIITA; RARRES2; GATM; KYNU; PDE1C; PTGER2; PLK3; ENDOU; PDIA3; NINJ2; CA2; ARHGDIG; PDE3B; CLEC4M; SLC7A10; PSMB8; NRAS; FGF21; CPB2; LHX2; EHD3; NREP; EIF2C4; GPR15; ZNF175;IL2; GNB3; FGFBP1; AMHR2; CALCR; CSF2; NFE2; NPPB; EPHX2; NOX3;CX3CL1; GIP; SAG; GAP43; ARL14; LMO2; C8B; NNMT; MAP1B; KLK8; GH2; GSTM3; CUX2; EBI3; NPBWR2; RSAD2; PGR; AVIL; SERPINA1; FMO3; CD300C; ORM1; IFIT3; RAMP3; RAB3B; KSR1; HTR2B; RAB25; PDPN; STAB1; NRP2; PLA2G7; RPGRIP1; HERC5; HSD17B2; RRH; FADS1; EMR1; LUM; STS; NODAL; NRCAM; HTR1B; NR5A2; NRG1; MT3; SULT1B1; IRAK3; GIMAP5; MICB; CNGA3; ABCB4; FES; PDGFRB; MAT1A; ACTN2; GNAI1; ARTN; APOA1; S100A7; CD8A; FZD9; IL6; DKK3; FZD5;P2RX6; IL15RA; RAC2; A2M; CACNA1H; REN; GULP1; IGFBP6; CD14;MIP; PRAME; ACSL5; SEMA3A; ZIC1; ARHGAP29; BIK; TRPC6; MPP1; CAMP; TRPM6; GPR77; GNA15

1.59E-08 VTN; PTPRM; DPYSL3; TIMP3; TNF; CD74; ACP5; CCL20; ABCA4; MYL9; BRCA2; LRP2; DLG4; ALDH3A1; STRA6; NTF3; CCL16; TH; NT5E; HRK; AKR1C3; TESC; SPARC; GHR; NGF; PTGS2; BMP7; KRT13; MGMT; SLC1A3; RARRES2; GATM; KYNU; NRAS; CPB2; LHX2; IL2; NOX3; CX3CL1; GIP; SAG; MAP1B; KLK8; PDPN; NRP2;PLA2G7; HSD17B2; RRH; FADS1; NRCAM; MT3; MICB; FES; PDGFRB; ARTN; APOA1; S100A7; IL6; RAC2;A2M; CACNA1H; ACSL5; SEMA3A; TRPC6; MPP1; GPR77 
Table III. Continued.

\begin{tabular}{|c|c|c|c|}
\hline GO ID & Term & P-value & Genes \\
\hline GO:0032501 & $\begin{array}{l}\text { Multicellular } \\
\text { organismal } \\
\text { process }\end{array}$ & $4.81 \mathrm{E}-08$ & $\begin{array}{l}\text { VTN; PTPRM; CXCR7; SNTB1; CCNA1; AKR1B10; LY6D; } \\
\text { ADAMTS9; DPYSL3; OLR1;TIMP3; DHX58; SPRR3; TFF3; TNF; CD14; } \\
\text { CNTNAP2; ODAM; ACP5; HEY2; ALOX5AP; PAQR5; POU3F2;TFF1; } \\
\text { IGF2BP3; ABCA4; MYL9; PTPRB; BRCA2; LRP2; DLG4; NME5; KCND2; } \\
\text { GUCY1B3; PRRX2; TBX2;CDH2; MAL; IL1R1; STRA6; NPC1L1; PPP1R9A; } \\
\text { NTF3; AFF3; SLC1A4; CDH11; TH; CELA3A; CELF3; TNNC1;ITGA6; NR2F1; } \\
\text { ESR1; CD3D; SRGN; AKR1C3; ACADL; LCAT; TAAR5; TESC; OLFM1; } \\
\text { CSPG4; SPARC; CUL3; INSL4; GPM6B; HSPB7; GHR; NGF; CEACAM1; } \\
\text { PTGS2; COLEC12; FOXA1; LEPREL1; BMP7; HOXC10; SIRPA; KRT13; } \\
\text { MGMT; BFSP2; SLC1A3; PCP4; FGD1; CIITA; SOAT2; RARRES2; GATM; } \\
\text { LMF1; NINJ2; PCDHA5; CA2; PDE3B; CLEC4M; SLC7A10; NRAS; FGF21; } \\
\text { CPB2; LHX2; EHD3; PCDHB12; NREP; IL2; ZNF287; GNB3; AMHR2; CSF2; } \\
\text { NFE2; NPPB; EPHX2; NOX3; CX3CL1; GIP; CRYAB; SAG; GAP43;LMO2; } \\
\text { KCNQ4; MAP1B; KLK8; CKMT2; GSTM3; EBI3; RSAD2; BARX2; AMELY; } \\
\text { PGR; AVIL; SERPINA1; LBX1; HOXA10; TNFAIP2; HTR2B; PDPN; } \\
\text { PCDHB11; STAB1; NRP2; PLA2G7;RPGRIP1; HERC5; CHODL; HSD17B2; } \\
\text { RRH; LUM; STS; NODAL; NRCAM; HTR1B; NR5A2; NRG1; MT3; CLGN; } \\
\text { IRAK3; GIMAP5; CNGA3; FES; PDGFRB; TFCP2L1; ACTN2; GNAI1; } \\
\text { ARTN; APOA1; S100A7; CD8A; FZD9; IL6; NEB; DKK3; NEUROD6; } \\
\text { FZD5; LIPC; P2RX6; IL15RA; RAC2; A2M; CACNA1H; REN; CAPN9;CD14; } \\
\text { MIP; SEMA3A; ZIC1; LAMC2; BIK; CSGALNACT1; TRPC6; GNA15; HAND1 }\end{array}$ \\
\hline
\end{tabular}

GO, gene ontology.

(http://www.geneontology.org/), and a pathway analysis based on the Kyoto Encyclopedia of Genes and Genomes (KEGG) database (http://www.genome.ad.jp/) contained in the functional analysis module of the INMEX program.

Analysis of protein-protein interaction (PPI) network. To determine the function of the proteins that they encoded, DEGs were imported into the PPI network constructed by using the Biological General Repository for Interaction Datasets (BioGRID) (http://thebiogrid.org/) in Cytoscape software (http://www.cytoscape.org/). The PPI network identified for the DEGs was screened at a genome-wide scale, with both end nodes having DEGs.

\section{Results}

Microarray datasets used in the meta-analysis. Three microarray datasets were found to meet our study criteria and these were extracted from the GEO database as a GEO series (GSE, an original record in GEO that summarizes an experiment). The datasets, GSE24460 and GSE3926, were microarray expression profiles of breast cancer cell lines that acquire drug resistance by stepwise treatment with doxorubicin. The other dataset, GSE54326, was a microarray expression profile of breast cancer cell lines that acquire drug resistance by stepwise treatment with epirubicin. As shown in Table I, from the 3 GSEs, we used 31 GEO samples (GSM, an identifier of specific experimental

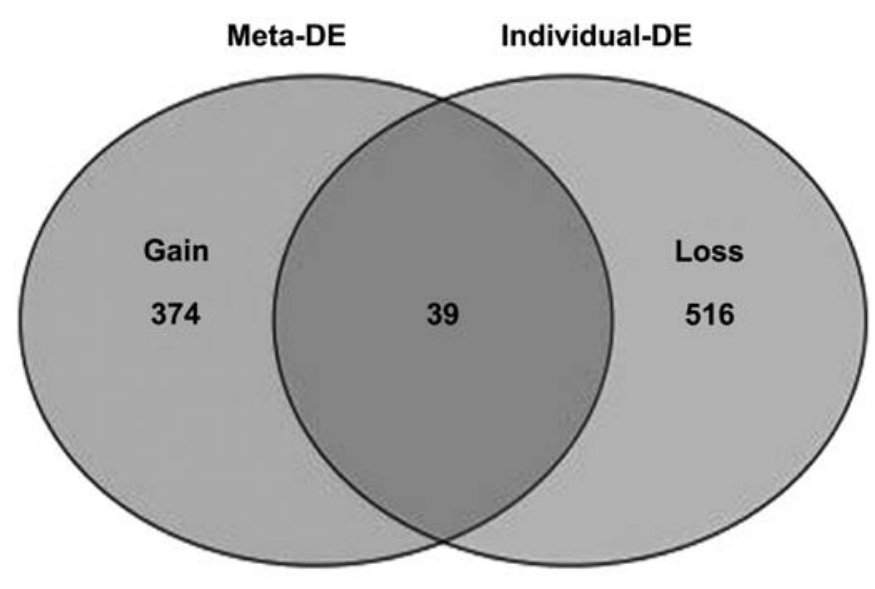

Figure 1. Venn diagram showing overlap between DEGs identified from the meta-analysis of multiple datasets (meta-DE) and the analysis of each individual dataset (individual-DE). DEGs, differentially expressed genes.

conditions) from 2 different GEO platforms (GPL, an identifier of specific microarray designs) in the meta-analysis.

Identification of DEGs commonly regulated in multiple datasets. We selected DEGs with $\mathrm{P}<0.05$ based on the estimated percentage of false-positives and P-values produced by the algorithm in RankProd. We identified 413 DEGs from GSMs in 


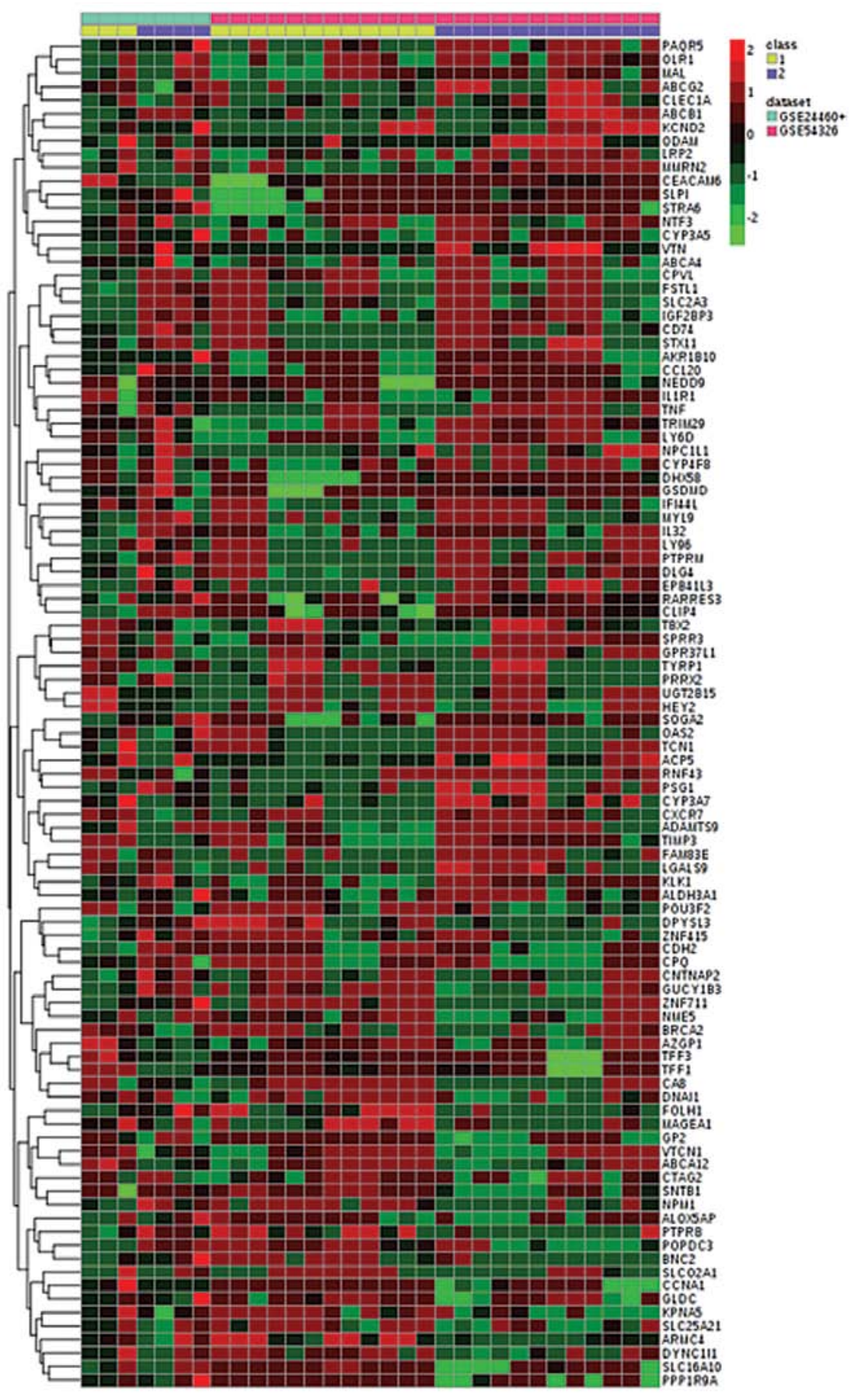

Figure 2. Heat-map representation of expression profiles for a particular DEG across different datasets by row-wise comparison. To prevent the problem dominated by study-specific effects, the map was regenerated by re-scaling individual datasets for visualization. Class 1 , parental control; class 2 , acquired anthracycline-resistant breast cancer. DEG, differentially expressed gene.

which acquired anthracycline-resistant breast cancer cell lines were compared with a parental control, including 255 up- and 158 downregulated genes. Additionally, 374 'gain' genes were identified in the meta-analysis, but not in any individual analysis (Fig. 1). The 20 most significantly up- or downregulated DEGs, with $\mathrm{P}<1.0 \mathrm{E}-5$, are shown in Table II. The upregulated genes were TRIM29, PTPRM, EPB4IL3, VTN, ABCBI, LY6D, ADAMTS9, OAS2, CXCR7, and AKR1B10. The downregulated genes were carbonic anhydrase VIII (CA8), ARMC4, SNTB1, CCNA1, karyopherin a 5 (KPNA5), POPDC3, ZNF711, FOLH1, SLC16A10, DPYSL3, TFF3, AZGP1, VTCN1, and
$C P V L$. Among these, the up- and downregulated DEGs with the largest mean $\log F C$ were TRIM29 and CA8, respectively (Table II). Heat maps, based on the meta-analysis of individual data sets, were used to visualize the correlation in expression patterns for a subset of genes from the three studies (Fig. 2).

Functional analysis of DEGs. The 413 DEGs were classified by GO biological processes. The most enriched terms of biological process were 'steroid metabolic process' (Table III). The KEGG pathway enrichment analysis was performed to select significantly overrepresented biochemical pathways 


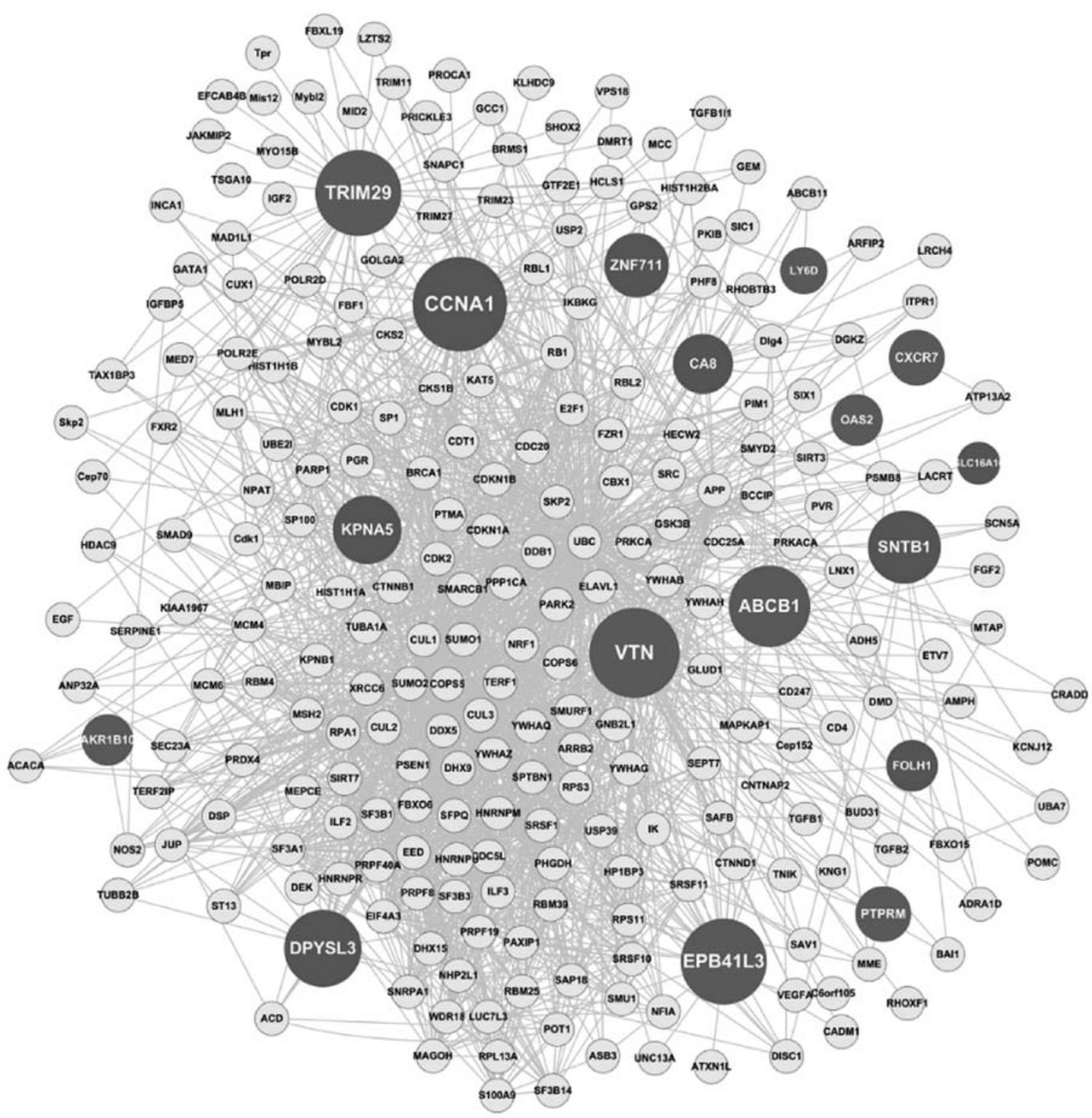

Figure 3. The protein-protein interaction networks of the top 10 up- and downregulated DEGs. The node and edge stand for protein (gene) and interaction, respectively. The size of the nodes represent the degree of interaction, suggesting that the bigger node the higher the degree of interaction. DEGs, differentially expressed genes.

(Table IV). Among the significantly enriched pathways (determined by a hypergeometric test, where $\mathrm{P}<0.05$ ), 'steroid hormone biosynthesis' was the most significant. Additionally, 'cytokine-cytokine receptor interaction' and 'drug metabolism-cytochrome P450' were highly enriched. The network of proteins encoded by the top 10 up- and downregulated DEGs were identified using the BioGRID PPI network (Fig. 3). The size of nodes representing proteins indicates the degree of interaction in the PPI, where larger nodes have more interactions. The proteins with significantly more interactions were encoded by the upregulated DEGs TRIM29, VTN, and $A B C B 1$, and the downregulated DEGs CCNA1 and KPNA5.

\section{Discussion}

A major obstacle in breast cancer chemotherapy is treatment failure due to anticancer drug resistance. Anthracyclines are one of the most commonly used chemotherapy agents in breast cancer; however, development of anthracycline resistance is a common limitation. In order to manage chemotherapy-resistant/refractory breast cancer, a comprehensive analysis of the mechanisms underlying the development of anthracycline resistance is essential. In the present study, we used publically available data sets and a meta-analysis approach, in which DEGs from various microarray datasets were combined and analyzed to identify genes that were consistently and significantly differentially expressed, to investigate the common biological signatures in the development of anthracycline resistance in breast cancer. Additionally, we investigated the DEGs by analysis of GO enrichment, KEGG pathways, and a constructed PPI network.

We identified 413 DEGs potentially involved in the development of anthracycline resistance. The upregulated gene with the most statistical significance was TRIM29 (tripartite motif containing 29), which is a member of the TRIM family, which is involved in hematologic and solid tumor cancers. It may 
Table IV. The top 15 KEGG pathway enrichment of the identified DEGs.

\begin{tabular}{llcc}
\hline KEGG ID & \multicolumn{1}{c}{ Pathway } & No. of genes & P-value \\
\hline hsa00140 & Steroid hormone biosynthesis & 9 & $1.91 \mathrm{E}-05$ \\
hsa04060 & Cytokine-cytokine receptor interaction & 15 & 0.00707 \\
hsa00982 & Drug metabolism - cytochrome P450 & 5 & 0.00811 \\
hsa00980 & Metabolism of xenobiotics by cytochrome P450 & 0.01756 \\
hsa00590 & Arachidonic acid metabolism & 6 & 0.03716 \\
hsa04726 & Serotonergic synapse & 5 & 0.04440 \\
hsa04540 & Gap junction & 6 & 0.04651 \\
hsa05145 & Toxoplasmosis & 6 & 0.05552 \\
hsa00040 & Pentose and glucuronate interconversions & 6 & 0.05704 \\
hsa05152 & Tuberculosis & 3 & 0.07015 \\
hsa00350 & Tyrosine metabolism & 9 & 0.07198 \\
hsa00260 & Glycine, serine and threonine metabolism & 3 & 0.07198 \\
hsa04810 & Regulation of actin cytoskeleton & 3 & 0.08736 \\
hsa04010 & MAPK signaling pathway & 9 & 0.08955 \\
hsa04612 & Antigen processing and presentation & 12 & 0.10430 \\
\hline
\end{tabular}

DEGs, differentially expressed genes; KEGG, Kyoto Encyclopedia of Genes and Genomes.

also function in the suppression of radiosensitivity because it is associated with the ataxia telangiectasia phenotype (19). Upregulation of TRIM29 reportedly promotes cancer cell proliferation and predicts poor survival in gastric, prostate and pancreatic cancer (19-21), however, its role with the development of anthracycline resistance has yet to be associated. By contrast, some of our DEGs had already linked to chemotherapeutic drug resistance. For example, consistent with previous studies, we found upregulation of the genes that encode proteins belonging to the multidrug resistance-associated protein (MRPs) family. MDR1/ABCB1 (or Pgp), a member of the $\mathrm{ABC}$ transporter superfamily, is a major contributor to resistance. A variety of Pgp inhibitors have been identified, but they show no consistent advantage in clinical studies $(8,9)$. The $A B C G 2$ gene encodes a unique member of the ABC halftransporter group that hydrolyzes ATP to efflux a large number of chemotherapeutic agents. The substrates of the ABCG2 protein include anticancer drugs primarily targeting topoisomerases, which include the anthracyclines (22). ABCG2-positive cells show increased tumorigenicity, and overexpression of ABCG2 enhances the capacity for proliferation and resistance to doxorubicin (23).

The most statistically significant downregulated DEG was $C A 8$. The protein encoded by this gene was initially termed 'CA-related protein' because of sequence homologues with other known carbonic anhydrase genes. However, $C A 8$ lacks carbonic anhydrase activity. Little is known with regard to how $C A 8$ functions in physiological processes, and its role has yet to be reported in relation to the development of drug resistance; therefore, it is a gene that remains to be investigated. KPNA5, also known as importin $\alpha 6$, was also identified as a significantly downregulated DEG. The KPNA5 protein belongs to the importin $\alpha$ protein family and is thought to be involved in nuclear localization signal (NLS)-dependent protein import into the nucleus. The mechanism underlying the acquisition of drug-resistance is probably linked to nuclear trafficking machinery. For example, the nuclear sparing phenomenon has been reported in drug resistant cells treated with various anthracyclines (24). Additionally, drug-sensitive cancer cells transport anthracyclines in their nuclei bound to a protein carrier (25). Therefore, the downregulation of nuclear trafficking-associated genes may contribute to the mechanism of anthracycline resistance.

In the GO term enrichment analysis of the 413 DEGs, enriched terms included the biological processes 'steroid metabolic process' and 'response to chemical and external stimuli', the molecular functions 'xenobiotic-transporting ATPase activity' and 'steroid dehydrogenase activity', and the cellular components 'plasma membrane' and 'extracellular region'. Of the 98 statistically significant pathways in our KEGG analysis, steroid hormone biosynthesis, cytokinecytokine receptor interaction, drug metabolism-cytochrome $\mathrm{P} 450$, and metabolism of xenobiotics by cytochrome P450 were the pathways most differentially regulated in relation to acquired anthracycline-resistant breast cancer. A number of drug efflux pumps are involved in the production and secretion of steroid hormone, and the expression is usually upregulated in tissues that partticipate in steroid hormone biosynthesis (26). Cytochrome P450s are enzymes that play a vital role in activating and inactivating many anticancer drugs, including anthracyclines (27). Therefore, cytochrome P450 pathways may be central to anthracycline resistance.

In our analysis, we identified a PPI network comprising the encoded proteins from the top 10 up- and downregulated DEGS. We found that TRIM29, ABCB1 and VTN were significant hub proteins in the upregulation of the PPI network, while CCNA1 and KPNA5 were hubs in the downregulation network. Taken together, our meta-analysis and PPI network strongly suggests that TRIM29 and KPNA5 are involved in the development of acquired anthracycline resistance in breast 
cancer. However, we acknowledge that further validation of the DEGs is required, and suggest that additional investigation could lead to the identification of new targets for anthracycline resistance and possibly the development of better cancer chemotherapy strategies.

In the present study, we followed a rigorous protocol for the systematic review, in which we comprehensively identified and analyzed data from three different databases. However, the results of our meta-analysis should be interpreted with caution in light of some unavoidable limitations. First, potential heterogeneity and confounding factors may have affected the analysis. For example, samples may be heterogeneous with respect to culture conditions, drug exposure time, drug concentrations, and microarray platforms. Second, ADR is a complex and multifactorial phenomenon, and thus potential gene-gene and gene-environment interactions must be considered. Despite these limitations, our meta-analysis, which is the most up-to-date review of the current evidence, provides a comprehensive view of gene expression patterns and new regulatory insight for acquired anthracycline-resistant breast cancer.

\section{Acknowledgements}

This study was supported by the National Research Foundation of Korea Grant funded by the Korean Government (NRF2013R1A1A1075999).

\section{References}

1. Langer SW, Sehested M and Jensen PB: Anthracycline extravasation: a comprehensive review of experimental and clinical treatments. Tumori 95: 273-282, 2009.

2. Gluck S: Adjuvant chemotherapy for early breast cancer: optimal use of epirubicin. Oncologist 10: 780-791, 2005.

3. Moreno-Aspitia A and Perez EA: Anthracycline- and/or taxane-resistant breast cancer: results of a literature review to determine the clinical challenges and current treatment trends. Clin Ther 31: 1619-1640, 2009.

4. Xu BH: Strategy in the treatment of anthracycline-resistant breast cancer. Zhonghua Zhong Liu Za Zhi 29: 241-244, 2007 (In Chinese).

5. Stavrovskaya AA: Cellular mechanisms of multidrug resistance of tumor cells. Biochemistry (Mosc) 65: 95-106, 2000.

6. Banerjee D, Mayer-Kuckuk P, Capiaux G, Budak-Alpdogan T, Gorlick R and Bertino JR: Novel aspects of resistance to drugs targeted to dihydrofolate reductase and thymidylate synthase. Biochim Biophys Acta 1587: 164-173, 2002.

7. Faneyte IF, Kristel PM and van de Vijver MJ: Multidrug resistance associated genes MRP1, MRP2 and MRP3 in primary and anthracycline exposed breast cancer. Anticancer Res 24: 2931-2939, 2004.
8. Liscovitch M and Lavie Y: Cancer multidrug resistance: a review of recent drug discovery research. IDrugs 5: 349-355, 2002.

9. van Zuylen L, Nooter K, Sparreboom A and Verweij J: Development of multidrug-resistance convertors: sense or nonsense? Invest New Drugs 18: 205-220, 2000.

10. Ravdin PM: Anthracycline resistance in breast cancer: clinical applications of current knowledge. Eur J Cancer 31A (Suppl 7): S11-S14, 1995.

11. Chien AJ and Moasser MM: Cellular mechanisms of resistance to anthracyclines and taxanes in cancer: intrinsic and acquired. Semin Oncol 35 (Suppl 2): S1-S14; quiz S39, 2008.

12. Gottesman MM: Mechanisms of cancer drug resistance. Annu Rev Med 53: 615-627, 2002.

13. Kang HC, Kim IJ, Park JH, et al: Identification of genes with differential expression in acquired drug-resistant gastric cancer cells using high-density oligonucleotide microarrays. Clin Cancer Res 10: 272-284, 2004.

14. Calcagno AM, Salcido CD, Gillet JP, et al: Prolonged drug selection of breast cancer cells and enrichment of cancer stem cell characteristics. J Natl Cancer Inst 102: 1637-1652, 2010.

15. Munkacsy G, Abdul-Ghani R, Mihaly Z, et al: PSMB7 is associated with anthracycline resistance and is a prognostic biomarker in breast cancer. Br J Cancer 102: 361-368, 2010.

16. Siddiqui AS, Delaney AD, Schnerch A, Griffith OL, Jones SJ and Marra MA: Sequence biases in large scale gene expression profiling data. Nucleic Acids Res 34: e83, 2006.

17. Moreau Y, Aerts S, De Moor B, De Strooper B and Dabrowski M: Comparison and meta-analysis of microarray data: from the bench to the computer desk. Trends Genet 19: 570-577, 2003.

18. Cohn LD and Becker BJ: How meta-analysis increases statistical power. Psychol Methods 8: 243-253, 2003.

19. Kosaka Y,Inoue H,Ohmachi T,etal: Tripartite motif-containing 29 (TRIM29) is a novel marker for lymph node metastasis in gastric cancer. Ann Surg Oncol 14: 2543-2549, 2007.

20. Wang L, Yang H, Palmbos PL, et al: ATDC/TRIM29 phosphorylation by ATM/MAPKAP kinase 2 mediates radioresistance in pancreatic cancer cells. Cancer Res 74: 1778-1788, 2014.

21. Kanno Y, Watanabe M, Kimura T, Nonomura K, Tanaka S and Hatakeyama S: TRIM29 as a novel prostate basal cell marker for diagnosis of prostate cancer. Acta Histochem 116: 708-712, 2014.

22. Dezi M, Fribourg PF, Di Cicco A, et al: The multidrug resistance half-transporter ABCG2 is purified as a tetramer upon selective extraction from membranes. Biochim Biophys Acta 1798: 2094-2101, 2010.

23. Zhang G, Wang Z, Luo W, Jiao H, Wu J and Jiang C: Expression of potential cancer stem cell marker ABCG2 is associated with malignant behaviors of hepatocellular carcinoma. Gastroenterol Res Pract 2013: 782581, 2013.

24. Featherstone JM, Speers AG, Lwaleed BA, Hayes MC, Cooper AJ and Birch BR: The nuclear membrane in multidrug resistance: microinjection of epirubicin into bladder cancer cell lines. BJU Int 95: 1091-1098, 2005

25. Kiyomiya K, Matsuo S and Kurebe M: Mechanism of specific nuclear transport of adriamycin: the mode of nuclear translocation of adriamycin-proteasome complex. Cancer Res 61: 2467-2471, 2001

26. Fujise H, Annoura T, Sasawatari S, Ikeda T and Ueda K: Transepithelial transport and cellular accumulation of steroid hormones and polychlorobiphenyl in porcine kidney cells expressed with human P-glycoprotein. Chemosphere 46: 1505-1511, 2002.

27. Doehmer J, Goeptar AR and Vermeulen NP: Cytochromes P450 and drug resistance. Cytotechnology 12: 357-366, 1993. 